\title{
max \\ On the Semi-Local Convergence of a Traub-Type Method for Solving Equations
}

\author{
Samundra Regmi ${ }^{1}$, , Christopher I. Argyros ${ }^{2}$, Ioannis K. Argyros ${ }^{3, *} *$ and Santhosh George ${ }^{4}(\mathbb{D}$ \\ 1 Learning Commons, University of North Texas at Dallas, Dallas, TX 75201, USA; \\ samundra.regmi@untdallas.edu \\ 2 Department of Computing and Technology, Cameron University, Lawton, OK 73505, USA; \\ christopher.Argyros@cameron.edu \\ 3 Department of Mathematical Sciences, Cameron University, Lawton, OK 73505, USA \\ 4 Department of Mathematical and Computational Sciences, National Institute of Technology Karnataka, \\ Mangaluru 575025, India; sgeorge@nitk.edu.in \\ * Correspondence: iargyros@cameron.edu
}

Citation: Regmi, S.; Argyros, C.I.; Argyros, I.K.; George, S. On the Semi-Local Convergence of a Traub-Type Method for Solving Equations. Foundations 2022, 2, 114-127. https://doi.org/10.3390/ foundations 2010006

Academic Editor: Vladimir Iosifovich Semenov

Received: 25 December 2021

Accepted: 12 January 2022

Published: 14 January 2022

Publisher's Note: MDPI stays neutral with regard to jurisdictional claims in published maps and institutional affiliations.

Copyright: (๑) 2022 by the authors Licensee MDPI, Basel, Switzerland. This article is an open access article distributed under the terms and conditions of the Creative Commons Attribution (CC BY) license (https:// creativecommons.org/licenses/by/ $4.0 /)$.

\begin{abstract}
The celebrated Traub's method involving Banach space-defined operators is extended. The main feature in this study involves the determination of a subset of the original domain that also contains the Traub iterates. In the smaller domain, the Lipschitz constants are smaller too. Hence, a finer analysis is developed without the usage of additional conditions. This methodology applies to other methods. The examples justify the theoretical results.
\end{abstract}

Keywords: Traub's method; Banach space; convergence criterion

MSC: 49M15; 47H17; 65J15; 65G99; 41A25

\section{Introduction}

The purpose of this article is to locate a solution $x^{*}$ of equation

$$
F(x)=0,
$$

provided that $F: \Omega \subset E_{1} \longrightarrow E_{2}$ is derivable according to Fréchet. Moreover, $E_{1}, E_{2}$ stand for Banach spaces, whereas $\Omega$ is nonempty and open.

The famous quadratically convergent Newton-Kantorovich method is defined for all $j=0,1,2, \ldots$ as

$$
x_{0} \in \Omega, x_{j+1}=x_{j}-F^{\prime}\left(x_{j}\right)^{-1} F\left(x_{j}\right)
$$

has been used extensively to produce sequence $\left\{x_{j}\right\}$ such that $\lim _{j \rightarrow \infty} x_{j}=x^{*}[1-8]$. Although there is a plethora on convergence results for (2) there exist some problems. In particular, the convergence ball is in general small [1-18]. Hence, it is important to extend this ball but with no additional conditions. Other defects relate to the accuracy of bounds on $\left\|x_{j+1}-x_{j}\right\|$ or $\left\|x_{j}-x^{*}\right\|$, as well as the results on the location uniqueness of $x^{*}$. The same defects appear in the study of high convergence order methods [19-22]. We have developed a technique that helps determine some $D \subseteq \Omega$, where iterates can also be found. This way, using $D$ instead of $\Omega$, a finer analysis is possible with no additional conditions.

We demonstrate our techniques for a certain high convergence order, although it can similarly be used on other methods [12,15-17].

We extend the two step Traub method [21] (see also [18,22]) to the following three step fifth order method 


$$
\begin{aligned}
y_{j} & =x_{j}-F^{\prime}\left(x_{j}\right)^{-1} F\left(x_{j}\right) \\
z_{j} & =y_{j}-F^{\prime}\left(x_{j}\right)^{-1} F\left(y_{j}\right) \\
x_{j+1} & =z_{j}-F^{\prime}\left(x_{j}\right)^{-1} F\left(z_{j}\right) .
\end{aligned}
$$

Traub's two-step method requires less computational effort than any third-order method utilizing the second derivative $[2,4,5,14]$.

Let us provide the earlier results.

(i) Convergence has been shown in Potra and Pták in [14] using

$$
\begin{gathered}
\left\|F^{\prime}\left(x_{0}\right)^{-1} F\left(x_{0}\right)\right\| \leq \mu \\
\left\|F^{\prime}\left(x_{0}\right)^{-1}\left(F^{\prime}(w)-F^{\prime}(v)\right)\right\| \leq L_{1}\|w-v\| \text { for all } w, v \in \Omega \\
T_{1}=2 L_{1} \mu \leq 1
\end{gathered}
$$

and

$$
U\left[x_{0}, \rho_{0}\right] \subset \Omega,
$$

and

$$
\rho_{0}=\frac{1-\sqrt{1-T_{1}}}{L_{1}},
$$

where $U\left[x_{0}, \rho_{0}\right]$ is a closed ball with radius $\rho_{0}$ and center at $x_{0} \in \Omega$. The centerLipschitz condition is introduced by us as

$$
\left\|F^{\prime}\left(x_{0}\right)^{-1}\left(F^{\prime}(w)-F^{\prime}\left(x_{0}\right)\right)\right\| \leq L_{0}\left\|w-x_{0}\right\| \text { for all } w \in \Omega .
$$

Define the set

$$
D=U\left(x_{0}, \frac{1}{L_{0}}\right) \cap \Omega .
$$

Moreover, we introduced the restricted-Lipschitz condition

$$
\left\|F^{\prime}\left(x_{0}\right)^{-1}\left(F^{\prime}(w)-F^{\prime}(v)\right)\right\| \leq L\|w-v\| \text { for all } w, v \in D
$$

However, then we notice that

$$
L_{0} \leq L_{1}
$$

and

$$
L \leq L_{1}
$$

hold, since

$$
D \subseteq \Omega
$$

Suppose

$$
T=2 L \mu \leq 1
$$

It follows that (9), (13), and

$$
\bar{\rho}_{0}=\frac{1-\sqrt{1-T}}{L}
$$

can used for (4), (5), and $\rho_{0}$, respectively, given in [14] (Theorem 5.2, p. 79). Hence,

$$
T_{1} \leq 1 \Rightarrow T \leq 1
$$

and

$$
\bar{\rho}_{0} \leq \rho_{0}
$$


hold. So, the applicability of Traub's method is extended. The parameters $L_{0}$ and $L$ are special cases of $L_{1}$, so no additional effort is used. It is also worth to mention that $L_{1}=L_{1}(\Omega), L_{0}=L_{0}(\Omega)$ but $L=L\left(\Omega, L_{0}\right)$. The proof in [14] (Theorem 5.2) utilized

$$
\left\|F^{\prime}\left(x_{0}\right)^{-1}\left(F^{\prime}(w)-F^{\prime}\left(x_{0}\right)\right)\right\| \leq L_{1}\left\|w-x_{0}\right\|<1
$$

leading to (by the Banach lemma [12] on linear invertible operators)

$$
\left\|F^{\prime}(w)^{-1} F^{\prime}\left(x_{0}\right)\right\| \leq \frac{1}{1-L_{1}\left\|w-x_{0}\right\|} .
$$

However, we get

$$
\left\|F^{\prime}\left(x_{0}\right)^{-1}\left(F^{\prime}(w)-F^{\prime}\left(x_{0}\right)\right)\right\| \leq L_{0}\left\|w-x_{0}\right\|<1
$$

leading to tighter

$$
\left\|F^{\prime}(w)^{-1} F^{\prime}\left(x_{0}\right)\right\| \leq \frac{1}{1-L_{0}\left\|w-x_{0}\right\|} .
$$

This modification in the proof brings the aforementioned advantages. In the numerical section one can find cases when (10)-(12) are strict.

(ii) In [12], they used

$$
\begin{gathered}
\left\|F^{\prime}\left(x_{0}\right)^{-1} F\left(x_{0}\right)\right\| \leq \mu, \\
\left\|F^{\prime}\left(x_{0}\right)^{-1} F^{\prime \prime}\left(x_{0}\right)\right\| \leq \tau, \\
\left\|F^{\prime}\left(x_{0}\right)^{-1}\left(F^{\prime \prime}(w)-F^{\prime \prime}(v)\right)\right\| \leq K_{1}\|w-v\| \text { for all } w, v \in \Omega, \\
\mu \leq \frac{\left(\tau^{2}+2 K_{1}\right)^{\frac{3}{2}}-\tau\left(\tau^{2}+3 K_{1}\right)}{3 K_{1}}
\end{gathered}
$$

and

$$
U\left[x_{0}, \rho_{1}\right] \subset \Omega,
$$

with $\rho_{1}$ denoting the minimal positive solution of equation

$$
\frac{K_{1}}{6} t^{3}+\frac{\tau}{2} t^{2}-t+\mu=0
$$

In our case we use

$$
\left\|F^{\prime}\left(x_{0}\right)^{-1}\left(F^{\prime \prime}(w)-F^{\prime \prime}(v)\right)\right\| \leq K\|w-v\| \text { for all } w, v \in D_{0},
$$

where

$$
D_{0}=U\left(x_{0}, \frac{1}{L_{0}}\right) \cap \Omega
$$

or

$$
D_{0}=U\left(x_{0}, \rho_{2}\right) \cap \Omega,
$$

if

$$
\left\|F^{\prime}\left(x_{0}\right)^{-1}\left(F^{\prime \prime}(w)-F^{\prime \prime}\left(x_{0}\right)\right)\right\| \leq K_{0}\left\|w-x_{0}\right\| \text { for all } w \in \Omega,
$$

is used, instead, where $\rho_{2}$ is the minimal positive solution of equation

$$
\frac{K}{6} t^{3}+\frac{\tau}{2} t^{2}-t+\mu=0
$$

Then, condition

$$
\mu \leq \frac{\left(\tau^{2}+2 K\right)^{\frac{3}{2}}-\tau\left(\tau^{2}+3 K\right)}{3 K}
$$


is the corresponding and weaker sufficient convergence criterion. Notice again that

$$
\begin{aligned}
& K \leq K_{1}, \\
& \rho_{2} \leq \rho_{1},
\end{aligned}
$$

$K_{1}=K_{1}(\Omega), K_{0}=K_{0}(\Omega)$, and $K=K\left(\Omega, K_{0}\right)$. The old estimate in [12] involving the bounds on $\left\|F^{\prime}(w)^{-1} F^{\prime}\left(x_{0}\right)\right\|$ is

$$
\left\|F^{\prime}(w)^{-1} F^{\prime}\left(x_{0}\right)\right\| \leq \frac{1}{1-\left(\tau\left\|w-x_{0}\right\|+\frac{K_{1}}{2}\left\|w-x_{0}\right\|^{2}\right)},
$$

whereas, we use

$$
\left\|F^{\prime}(w)^{-1} F^{\prime}\left(x_{0}\right)\right\| \leq \frac{1}{1-\left(\tau\left\|w-x_{0}\right\|+\frac{K}{2}\left\|w-x_{0}\right\|^{2}\right)},
$$

which is more precise.

The rest of the paper is organized as follows: Next, Section 2 contains the convergence analysis, whereas the numerical examples and conclusions can be found in Sections 3 and 4, respectively.

\section{Majorizing Sequences}

We introduce some auxiliary results on scalar majorizing sequences.

Definition 1. Let $\left\{p_{j}\right\}$ be a Banach space valued sequence. Then, a nondecreasing scalar sequence $\left\{q_{j}\right\}$ is majorizing for $\left\{p_{j}\right\}$, if

$$
\left\|p_{j+1}-p_{j}\right\| \leq q_{j+1}-q_{j} \text { for each } j=0,1,2, \ldots
$$

So, the convergence of sequence $\left\{p_{j}\right\}$ reduces to studying that of $\left\{q_{j}\right\}$ [14]. Set $H=[0, \infty)$ and $H_{0}=[0, b)$ for some $b>0$.

Let $\mu \geq 0$ be a parameter and $\varphi_{0}: H \longrightarrow H, \varphi: H_{0} \longrightarrow H$ be continuous and non-decreasing function. We shall use scalar sequences $\left\{t_{j}\right\},\left\{s_{j}\right\}$ and $\left\{u_{j}\right\}$ defined for each $j=0,1,2, \ldots$ by $t_{0}=0, s_{0}=\mu$

$$
\begin{aligned}
u_{j} & =s_{j}+\frac{\int_{0}^{1} \bar{\varphi}\left((1-\theta)\left(s_{j}-t_{j}\right)\right) d \theta\left(s_{j}-t_{j}\right)}{1-\varphi_{0}\left(t_{j}\right)} \\
t_{j+1} & =u_{j}+\frac{\int_{0}^{1} \bar{\varphi}\left(s_{j}-t_{j}+\theta\left(u_{j}-s_{j}\right)\right) d \theta\left(u_{j}-s_{j}\right)}{1-\varphi_{0}\left(t_{j}\right)} \\
s_{j+1} & =t_{j+1}+\frac{\int_{0}^{1} \bar{\varphi}\left(u_{j}-t_{j}+\theta\left(t_{j+1}-u_{j}\right)\right) d \theta\left(t_{j+1}-u_{j}\right)}{1-\varphi_{0}\left(t_{j+1}\right)},
\end{aligned}
$$

where $\bar{\varphi}=\left\{\begin{array}{cc}\varphi_{0}, & j=0 \\ \varphi, & j=1,2, \ldots\end{array}\right.$

Next, we present a convergence result for a sequence $\left\{t_{j}\right\}$ under very general conditions

Lemma 1. Suppose:

(a) for all $j=0,1,2, \ldots$

$$
\varphi_{0}\left(t_{j}\right)<1
$$

and

$$
t_{j} \leq a \text { for some } a>0 .
$$


(b) Function $\varphi_{0}: H \longrightarrow H$ is continuous, increasing and

$$
t_{j} \leq \varphi_{0}^{-1}(1)
$$

for each $j=0,1,2, \ldots$ Then, sequences $\left\{t_{j}\right\},\left\{s_{j}\right\},\left\{u_{j}\right\}$ converge monotonically to $s_{* *}$, which is their unique upper bound (least).

Proof. (a) By (17)-(19): $0 \leq t_{j} \leq s_{j} \leq u_{j} \leq t_{j+1}$, are bounded by $a$ so, they converge to $s_{*} \in[\mu, a]$.

(b) By (17) and (20) one has again

$$
\lim _{j \rightarrow \infty} t_{j}=\lim _{j \rightarrow \infty} s_{j}=\lim _{j \longrightarrow \infty} u_{j}=s_{*} \cdot
$$

Remark 1. Conditions (18)-(20) can be replaced by stronger, which however are easier to satisfy. This is why we give alternative criteria (but stronger) that can easier be verified.

We introduce, sequences functions and sequences of functions as follows

$$
\begin{aligned}
& a_{j}=\frac{\int_{0}^{1} \bar{\varphi}\left((1-\theta)\left(s_{j}-t_{j}\right)\right) d \theta}{1-\varphi_{0}\left(t_{j}\right)}, \\
& b_{j}=\frac{\int_{0}^{1} \bar{\varphi}\left(u_{j}-t_{j}+\theta\left(u_{j}-s_{j}\right)\right) d \theta}{1-\varphi_{0}\left(t_{j}\right)}, \\
& c_{j}=\frac{\int_{0}^{1} \bar{\varphi}\left(u_{j}-t_{j}+\theta\left(t_{j+1}-u_{j}\right)\right) d \theta}{1-\varphi_{0}\left(t_{j+1}\right)}, \\
& \bar{h}_{j}^{(1)}(t)=\int_{0}^{1} \varphi\left((1-\theta)\left(s_{j}-t_{j}\right)\right) d \theta+t \varphi_{0}\left(t_{j}\right)-t, \\
& h_{j}^{(1)}(t)=\int_{0}^{1} \varphi\left((1-\theta) t^{j} \mu\right) d \theta \\
& +t \varphi_{0}\left(\frac{1-t^{j+1}}{1-t} \mu\right)-t \\
& h^{(1)}(t)=\int_{0}^{1} \varphi((1-\theta) \mu) d \theta+t \varphi_{0}\left(\frac{\mu}{1-t}\right)-t \text {, } \\
& \bar{h}_{j}^{(2)}(t)=\int_{0}^{1} \varphi\left(s_{j}-t_{j}+\theta\left(u_{j}-s_{j}\right)\right) d \theta+t \varphi_{0}\left(t_{j}\right)-t, \\
& h_{j}^{(2)}(t)=\int_{0}^{1} \varphi\left((1+\theta t) t^{2 j} \mu\right) d \theta \\
& +t \varphi_{0}\left(\frac{1-t^{2 j+1}}{1-t} \mu\right)-t \\
& h^{(2)}(t)=\int_{0}^{1} \varphi((1+\theta t) \mu) d \theta+t \varphi_{0}\left(\frac{\mu}{1-t}\right)-t, \\
& \bar{h}_{j}^{(3)}(t)=\int_{0}^{1} \varphi\left(u_{j}-t_{j}+\theta\left(t_{j+1}-u_{j}\right)\right) d \theta+t \varphi_{0}\left(t_{j+1}\right)-t, \\
& h_{j}^{(3)}(t)=\int_{0}^{1} \varphi\left(\left(1+t+\theta t^{2}\right) t^{2 j} \mu d \theta+t \varphi_{0}\left(\frac{1-t^{2 j+3}}{1-t} \mu\right)-t\right.
\end{aligned}
$$


and

$$
h^{(3)}(t)=\int_{0}^{1} \varphi\left(\left(1+t+\theta t^{2}\right) \mu\right) d \theta+t \varphi_{0}\left(\frac{\mu}{1-t}\right)-t .
$$

Next, we present a second convergence result for $\left\{t_{j}\right\}$.

Lemma 2. Suppose:

There exists parameter $\gamma \in[0,1)$ such that

$$
\begin{gathered}
0 \leq a_{0} \leq \gamma, 0 \leq b_{0} \leq \gamma, 0 \leq c_{0} \leq \gamma, \\
0 \leq \varphi_{0}\left(t_{1}\right)<1, \\
h^{(1)}(\gamma) \leq 0, h^{(2)}(\gamma) \leq 0 \text { and } h^{(3)}(\gamma) \leq 0 .
\end{gathered}
$$

Then, sequences $\left\{t_{j}\right\},\left\{s_{j}\right\},\left\{u_{j}\right\}$ converge to $s_{*} \in\left[\mu, s_{* *}\right]$, where $s_{* *}=\frac{1}{1-\gamma} \mu$. Moreover, the following estimates hold

$$
\begin{gathered}
0 \leq s_{j}-t_{j} \leq \gamma^{j}\left(t_{j}-s_{j-1}\right) \leq \gamma^{2 j} \mu, \\
0 \leq u_{j}-s_{j} \leq \gamma\left(s_{j}-t_{j}\right) \leq \gamma^{2 j+1} \mu, \\
0 \leq t_{j+1}-u_{j} \leq \gamma\left(u_{j}-s_{j}\right) \leq \gamma^{2 j+2} \mu, \\
t_{j+1} \leq \frac{1-\gamma^{2 j+3}}{1-\gamma} \mu,
\end{gathered}
$$

and

$$
t_{j} \leq s_{j} \leq u_{j} \leq t_{j+1} \leq s_{* *} .
$$

Furthermore, we have $\varphi_{0}\left(t_{j}\right)<1$ for each $j=0,1,2, \ldots$.

Proof. Estimates (24)-(28) hold if

$$
\begin{aligned}
& 0 \leq a_{m} \leq \gamma \\
& 0 \leq b_{m} \leq \gamma \\
& 0 \leq c_{m} \leq \gamma \\
& \varphi_{0}\left(t_{m+1}\right)<1
\end{aligned}
$$

and

$$
t_{m} \leq s_{m} \leq u_{m} \leq t_{m+1}
$$

hold for each $m=0,1,2, \ldots$ However, they are true for $m=0$, by (21)-(23). Notice that we have by the definition (17) and these conditions that

$$
\begin{aligned}
t_{m+1} & \leq u_{m}+\gamma^{2 m+2} \mu \leq s_{m}+\gamma^{2 m+1} \mu+\gamma^{2 m+2} \mu \\
& \leq t_{m}+\gamma^{2 m} \mu+\gamma^{2 m+1} \mu+\gamma^{2 m+2} \mu \\
& \cdots \mu+\gamma \mu+\ldots+\gamma^{2 m+2} \mu \\
& =\frac{1-\gamma^{2 m+3}}{1-\gamma} \mu \leq \frac{\mu}{1-\gamma}=s_{* *} .
\end{aligned}
$$

Suppose, estimates (24)-(28) hold for all integers smaller or equal to $k$. Hence, by replacing $t_{0}, s_{0}, u_{0}, t_{1}$ by $t_{m}, s_{m}, u_{m}, t_{m+1}$ and using the induction hypotheses we see that (29)-(33) shall be true if

$$
\bar{h}_{j}(\gamma)^{(i)} \leq 0
$$


or

$$
h_{j}(\gamma)^{(i)} \leq 0
$$

or

$$
h(\gamma)^{(i)} \leq 0,
$$

for $i=1,2,3$ and $j=0,1,2, \ldots, m$, which holds true by (23). The induction for (24)-(28) is terminated. The remaining of the proof can be found in Lemma 1.

Remark 2. (a) The conditions of Lemma 2 imply those of Lemma 1 but not necessarily vice versa.

(b) Consider functions " $\varphi$ " to be given by $\varphi_{0}$ and $\varphi$ in the interesting case $\varphi_{0}(t)=L_{0} t$ and $\varphi(t)=L t$ for $L_{0}>0$, and $L>0$.

Then, consider functions $f_{j}^{(i)}$ on $[0,1)$ given by

$$
\begin{aligned}
f_{j}^{(1)}(t)= & \frac{1}{2} t^{2 j-1} \mu \\
& +L_{0}\left(1+t+\ldots+t^{2 j}\right) \mu-1, \\
f_{j}^{(2)}(t)= & L\left(1+\frac{1}{2} t\right) t^{2 j-1} \mu \\
& +L_{0}\left(1+t+\ldots+t^{2 j}\right) \mu-1, \\
f_{j}^{(3)}(t)=\quad & \\
& +L_{0}\left(1+t+\frac{t^{2}}{2}\right) t^{2 j-1} \mu \\
g_{1}(t)= & L_{0} t^{3}+\left(L_{0}+\frac{L}{2}\right) t^{2}-\frac{L}{2} \\
g_{2}(t)=\left(L_{0}+\right. & \left.\frac{L}{2}\right) t^{3}+\left(L_{0}+L\right) t^{2}-\frac{L}{2} t-L
\end{aligned}
$$

and

$$
g_{3}(t)=L_{0} t^{5}+\left(L_{0}+\frac{L}{2}\right) t^{4}+L t^{3}+\frac{L}{2} t^{2}-L t-L .
$$

By these definitions, we have

$$
\begin{aligned}
& g_{1}(0)=-\frac{L}{2}<0, g_{1}(1)=2 L_{0}>0, \\
& g_{2}(0)=-L, g_{2}(1)=2 L_{0} \\
& g_{3}(0)=-L \text { and } g_{3}(1)=2 L_{0} .
\end{aligned}
$$

It follows from the intermediate value theorem (IVT) that functions $g_{i}$ have zeros in $(0,1)$. Denote the minimal such zeros by $\gamma_{i}$, respectively.

Define parameters

$$
\lambda_{0}=\max \left\{a_{0}, b_{0}, c_{0}\right\}, \lambda_{1}=\min \left\{\gamma_{1}, \gamma_{2}, \gamma_{3}\right\}
$$

and

$$
\lambda_{2}=\max \left\{\gamma_{1}, \gamma_{2}, \gamma_{3}\right\} .
$$

Then, we can show a third result on the convergence of sequence $\left\{t_{j}\right\}$.

Lemma 3. Suppose:

There exists $\gamma \in[0,1)$ satisfying

$$
\lambda_{0} \leq \lambda_{1} \leq \gamma \leq \lambda_{2}<1-L_{0} \mu .
$$


Then, the conclusions of Lemma 2 for a sequence $\left\{t_{j}\right\}$ follow.

Proof. We must show by Lemma 2

$$
h_{m}^{(i)}(\gamma) \leq 0
$$

But by the preceding definitions, we can show instead

$$
f_{m}^{(i)}(\gamma) \leq 0
$$

We must relate $f_{m+1}^{(i)}(t)$ to $f_{m}^{(i)}$. We can write

$$
\begin{aligned}
f_{m+1}^{(i)}(t)= & \frac{L}{2} t^{2 m+1}+L_{0}\left(1+t+\ldots+t^{2 m+2}\right) \mu-1 \\
& -\frac{L}{2} t^{2 m-1} \mu-L_{0}\left(1+t+\ldots+t^{2 m}\right) \mu+1+f_{m}^{(1)}(t) \\
= & f_{m}^{(1)}(t)+\left(\frac{L}{2} t^{2}+L_{0}\left(t^{2}+t^{3}\right)-\frac{L}{2}\right) t^{2 m-1} \mu \\
= & f_{m}^{(1)}(t)+g_{1}(t) t^{2 m-1} \mu,
\end{aligned}
$$

so

$$
f_{m+1}^{(1)}(t)=f_{m}^{(1)}(t)+g_{1}(t) t^{2 m-1} \mu .
$$

In particular, by (38) and the definition of $\gamma$

$$
f_{m+1}^{(1)}(\gamma) \leq f_{m}^{(1)}(\gamma)
$$

Define function $f_{\infty}^{(1)}$ by

$$
f_{\infty}^{(1)}(t)=\lim _{m \longrightarrow \infty} f_{m}^{(1)}(t) .
$$

Then, we have by (40)

$$
f_{\infty}^{(1)}(t)=\frac{L_{0} \mu}{1-t}-1
$$

So, we can show instead of (37) (for $i=1$ ) that

$$
f_{\infty}^{(1)}(\gamma) \leq 0
$$

which is true by (35). Similarly, we get

$$
\begin{aligned}
f_{m+1}^{(2)}(t)= & L\left(1+\frac{t}{2}\right) t^{2 m+1} \mu+L_{0}\left(1+t+\ldots+t^{2 m+2}\right) \mu-1 \\
& -L\left(1+\frac{t}{2}\right)\left(1+\frac{t}{2}\right) t^{2 m-1} \mu-L_{0}\left(1+t+\ldots+t^{2 m}\right)+1+f_{m}^{(2)}(t) \\
= & f_{m}^{(2)}(t)+\left[\left(1+\frac{t}{2}\right) L t^{2}-\left(1+\frac{t}{2}\right) L+L_{0}\left(t^{2}+t^{3}\right)\right] t^{2 m-1} \mu \\
= & f_{m}^{(2)}(t)+g_{2}(t) t^{2 m-1} \mu
\end{aligned}
$$

so

$$
f_{m+1}^{(2)}(t)=f_{m}^{(2)}(t)+=g_{2}(t) t^{2 m-1} \mu .
$$

In particular, we have

$$
f_{m+1}^{(2)}(\gamma) \leq f_{m}^{(2)}(\gamma)
$$

and again

$$
f_{\infty}^{(2)}(t)=\frac{L_{0} \mu}{1-t}-1
$$


Therefore, (37) (for $i=2$ ) reduces to showing

$$
f_{\infty}^{(2)}(\gamma) \leq 0
$$

which is true by (35). Moreover, we have analogously

$$
\begin{aligned}
f_{m+1}^{(3)}(t)= & L\left(1+t+\frac{t^{2}}{2}\right) t^{2 m+1} \mu+L_{0}\left(1+t+\ldots+t^{2 m+1}\right) \mu-1 \\
& -L\left(1+t+\frac{t^{2}}{2}\right) t^{2 m-1} \mu-L_{0}\left(1+t+\ldots+t^{2 m+2}\right) \mu+1+f_{m}^{(3)}(t) \\
= & f_{m}^{(3)}(t)+\left[L\left(1+t+\frac{t^{2}}{2}\right) t^{2}\right. \\
& \left.+L_{0}\left(t^{4}+t^{5}\right)-L\left(1+t+\frac{t^{2}}{2}\right)\right] t^{2 m-1} \mu \\
= & f_{m}^{(3)}(t)+g_{3}(t) t^{2 m-1} \mu,
\end{aligned}
$$

so

$$
f_{m+1}^{(3)}(\gamma) \leq f_{m}^{(3)}(\gamma)
$$

Hence, we can show instead of (37) (for $i=3$ ) that

$$
f_{\infty}^{(3)}(\gamma) \leq 0
$$

which is true by (34), where

$$
f_{\infty}^{(3)}(t)=\lim _{m \longrightarrow \infty} f_{m}^{(3)}(t)=\frac{L_{0} \mu}{1-t}-1
$$

Therefore, sequence $\left\{t_{j}\right\}$ is nondecreasing and bounded from above by $s_{* *}=\frac{1}{1-\gamma} \mu$, so it converges to $s_{*}$.

Next, we connect Lemmas 1 and 2 to method (3). We first consider conditions (A): Suppose

(A1) There exists $x_{0} \in \Omega, \mu \geq 0$ such that $F^{\prime}\left(x_{0}\right)^{-1} \in L\left(E_{2}, E_{1}\right)$ and

$$
\left\|F^{\prime}\left(x_{0}\right)^{-1} F\left(x_{0}\right)\right\| \leq \mu \text {. }
$$

(A2) For all $w \in \Omega$

$$
\left\|F^{\prime}\left(x_{0}\right)^{-1}\left(F^{\prime}(w)-F^{\prime}\left(x_{0}\right)\right)\right\| \leq \varphi_{0}\left(\left\|w-x_{0}\right\|\right) .
$$

(A3) Function $\varphi_{0}(t)-1$ has a smallest positive solution $\rho$. Set

$$
\Omega_{0}=U\left(x_{0}, \rho\right) \cap \Omega \text {. }
$$

(A4) For each $w, v \in \Omega_{0}$

$$
\left\|F^{\prime}\left(x_{0}\right)^{-1}\left(F^{\prime}(w)-F^{\prime}(v)\right)\right\| \leq \varphi(\|w-v\|)
$$

(A5) Hypotheses of Lemma 1 or Lemma 2 or Lemma 3 hold and (A6) $U\left[w_{0}, s_{*}\right] \subset \Omega$ (or $U\left[w_{0}, s_{* *}\right] \subset \Omega$ ).

Next, we prove the first semi-local convergence theorem for sequence $\left\{w_{j}\right\}$.

Theorem 1. Suppose hypotheses (A) hold. Then, sequences $\left\{w_{j}\right\}$ produced by method (3) is well defined in $U\left[w_{0}, s_{*}\right]$, remains in $U\left[w_{0}, s_{*}\right]$ for each $j=0,1,2, \ldots$ and converges to a solution $w_{*} \in U\left[w_{0}, s_{*}\right]$ (or $w_{*} \in U\left[w_{0}, s_{* *}\right]$ ) of equation $F(w)=0$. 
Proof. Using condition (A1) and the first substep of method (3) for $j=0$, we see that $y_{0}$ is well defined and

$$
\left\|y_{0}-x_{0}\right\|=\left\|F^{\prime}\left(x_{0}\right)^{-1} F\left(x_{0}\right)\right\| \leq \mu=s_{0}-t_{0}=s_{0} \leq s_{*},
$$

so $y_{0} \in U\left(x_{0}, s_{*}\right)$. Iterate $z_{0}$ is exists by (A1) and (3) for $j=0$. So, by (3) and (A3) one has

$$
\begin{aligned}
\left\|z_{0}-y_{0}\right\| & =\left\|F^{\prime}\left(x_{0}\right)^{-1} F\left(y_{0}\right)\right\| \\
& =\left\|F^{\prime}\left(x_{0}\right)^{-1}\left(F\left(y_{0}\right)-F\left(x_{0}\right)-F^{\prime}\left(x_{0}\right)\left(y_{0}-x_{0}\right)\right)\right\| \\
& =\left\|\int_{0}^{1} F^{\prime}\left(x_{0}\right)^{-1}\left(F^{\prime}(x)-F^{\prime}\left(x_{0}+\theta\left(y_{0}-x_{0}\right)\right)\right) d \theta\left(y_{0}-x_{0}\right)\right\| \\
& \leq \int_{0}^{1} \bar{\varphi}\left((1-\theta)\left\|y_{0}-x_{0}\right\|\right) d \theta\left\|y_{0}-x_{0}\right\| \\
& \leq \int_{0}^{1} \bar{\varphi}\left((1-\theta)\left(s_{0}-t_{0}\right)\right) d \theta\left(s_{0}-t_{0}\right) \leq u_{0}-s_{0} .
\end{aligned}
$$

We also have $\left\|z_{0}-x_{0}\right\| \leq\left\|z_{0}-y_{0}\right\|+\left\|y_{0}-x_{0}\right\| \leq u_{0}-s_{0}+s_{0}-t_{0}=u_{0}-t_{0}<s_{*}$, so $z_{0} \in U\left(x_{0}, s_{*}\right)$. By condition (A1) and (3) for $j=0, x_{1}$ exists, and we can write

$$
\begin{aligned}
x_{1}-y_{0} & =z_{0}-x_{0}-F^{\prime}\left(x_{0}\right)^{-1}\left(F\left(z_{0}\right)-F\left(x_{0}\right)\right) \\
& =F^{\prime}\left(x_{0}\right)^{-1} \int_{0}^{1}\left[F^{\prime}\left(x_{0}\right)-F^{\prime}\left(x_{0}+\theta\left(z_{0}-x_{0}\right)\right)\right] d \theta\left(z_{0}-x_{0}\right) .
\end{aligned}
$$

The condition (A2) and (17) give in turn that

$$
\begin{aligned}
\left\|x_{1}-y_{0}\right\| & \leq \int_{0}^{1} \varphi_{0}\left((1-\theta)\left\|z_{0}-x_{0}\right\|\right) d \theta\left\|z_{0}-x_{0}\right\| \\
& \leq \frac{\int_{0}^{1} \varphi_{0}\left((1-\theta)\left\|z_{0}-x_{0}\right\|\right) d \theta\left\|z_{0}-x_{0}\right\|}{1-\varphi_{0}\left(\left\|x_{0}-x_{0}\right\|\right)} \\
& \leq \frac{\int_{0}^{1} \varphi_{0}\left((1-\theta)\left(u_{0}-t_{0}\right)\right) d \theta\left(u_{0}-t_{0}\right)}{1-\varphi_{0}\left(t_{0}\right)} \\
& =t_{1}-s_{0} .
\end{aligned}
$$

We also have

$$
\begin{aligned}
\left\|x_{1}-x_{0}\right\| & \leq\left\|x_{1}-y_{0}\right\|+\left\|y_{0}-x_{0}\right\| \\
& \leq t_{1}-s_{0}+s_{0}-t_{0}=t_{1} \leq s_{*}
\end{aligned}
$$

so $w_{1} \in U\left(w_{0}, s_{*}\right)$. Let $w \in U\left(w_{0}, s_{*}\right)$. Using (A2) one obtains

$$
\left\|F^{\prime}\left(w_{0}\right)^{-1}\left(F^{\prime}(w)-F^{\prime}\left(w_{0}\right)\right)\right\| \leq \varphi_{0}\left(\left\|w-w_{0}\right\|\right) \leq \varphi_{0}\left(s_{*}\right)<1,
$$

so the Banach lemma for linear invertible operators [5] assures the existence of $F^{\prime}(w)^{-1}$ and

$$
\left\|F^{\prime}(w)^{-1} F^{\prime}\left(w_{0}\right)\right\| \leq \frac{1}{1-\varphi_{0}\left(\left\|w-w_{0}\right\|\right)} .
$$


In particular for $w=w_{1}, F^{\prime}\left(w_{1}\right)^{-1}$ exists, so does iterate $y_{1}$. Then, we can write by the first substep of method (3) for $k=1$

$$
\begin{aligned}
\left\|y_{1}-x_{1}\right\| & =\left\|F^{\prime}\left(x_{1}\right)^{-1} F\left(x_{1}\right)\right\| \\
& \leq\left\|F^{\prime}\left(x_{1}\right)^{-1} F^{\prime}\left(x_{0}\right)\right\|\left\|F^{\prime}\left(x_{0}\right)^{-1} F\left(x_{1}\right)\right\| \\
& \leq \frac{\left\|\int_{0}^{1} F^{\prime}\left(x_{0}\right)^{-1}\left(F^{\prime}\left(z_{0}+\theta\left(x_{1}-z_{0}\right)\right)-F^{\prime}\left(x_{0}\right)\right) d \theta\left(x_{1}-z_{0}\right)\right\|}{1-\varphi_{0}\left(\left\|x_{1}-x_{0}\right\|\right)} \\
& \leq \frac{\int_{0}^{1} \varphi_{0}\left(\left\|z_{0}-x_{0}\right\|+\theta\left\|x_{1}-z_{0}\right\|\right) d \theta\left\|x_{1}-z_{0}\right\|}{1-\varphi_{0}\left(\left\|x_{1}-x_{0}\right\|\right)} \\
& \leq \frac{\int_{0}^{1} \bar{\varphi}\left(u_{0}-t_{0}+\theta\left(t_{1}-u_{0}\right)\right) d \theta\left(t_{1}-u_{0}\right)}{1-\varphi_{0}\left(t_{1}\right)}
\end{aligned}
$$

where we also used by the definition of the method

$$
\begin{aligned}
\left\|F^{\prime}\left(x_{0}\right)^{-1} F\left(x_{1}\right)\right\| & =\| F^{\prime}\left(x_{0}\right)^{-1}\left(F\left(x_{1}\right)-F\left(z_{0}\right)+F\left(z_{0}\right)\right) \\
& \leq \int_{0}^{1} F^{\prime}\left(x_{0}\right)^{-1}\left(F^{\prime}\left(z_{0}+\theta\left(x_{1}-z_{0}\right)\right)-F^{\prime}\left(x_{0}\right)\right) d \theta\left(x_{1}-z_{0}\right) \| \\
& \leq \int_{0}^{1} \varphi_{0}\left(\left\|z_{0}-x_{0}\right\|+\theta\left\|x_{1}-z_{0}\right\|\right) d \theta\left\|x_{1}-z_{0}\right\| \\
& \leq \int_{0}^{1} \bar{\varphi}\left(u_{0}-t_{0}+\theta\left(t_{1}-u_{0}\right)\right) d \theta\left(t_{1}-u_{0}\right) \\
& =s_{1}-t_{1},
\end{aligned}
$$

and

$$
\left\|x_{1}-x_{0}\right\| \leq\left\|x_{1}-y_{0}\right\|+\left\|y_{0}-x_{0}\right\| \leq t_{1}-s_{0}+s_{0}-t_{0}=t_{1}-t_{0} .
$$

Hence, we showed so far

$$
\begin{gathered}
\left\|y_{m}-x_{m}\right\| \leq s_{m}-t_{m}, m=0,1, \\
\left\|z_{m}-y_{m}\right\| \leq u_{m}-s_{m}, m=0, \\
\left\|x_{m+1}-z_{m}\right\| \leq t_{m+1}-u_{m}, \quad m=0,
\end{gathered}
$$

and

$$
x_{m}, y_{m}, z_{m}, x_{m+1} \in U\left(x_{0}, s_{*}\right)
$$

for $m=0$. Consider these estimates are true for all $m \leq j-1$. Then, simply replace $x_{0}, y_{0}, z_{0}, x_{1}$ by $x_{m}, y_{m}, z_{m}, x_{m+1}$, to terminate the induction for items (46)-(49). So, $\left\{x_{j}\right\}$ is fundamental in a Banach space $E_{1}$. Hence, $\lim _{j \rightarrow \infty} x_{j}=x^{*} \in U\left[x_{0}, s_{*}\right]$. Then, by letting $j \longrightarrow \infty$ in the estimate (see also (46))

$$
\left\|F^{\prime}\left(x_{0}\right)^{-1} F\left(x_{j+1}\right)\right\| \leq \int_{0}^{1} \varphi\left(u_{j}-t_{j}+\theta\left(t_{j+1}-u_{j}\right)\right) d \theta\left(t_{j+1}-u_{j}\right),
$$

and the continuity of $F$, we conclude $F\left(x_{*}\right)=0$.

A uniqueness of the solution result follows.

Proposition 1. Suppose:

(i) There exists a simple solution $x^{*} \in \Omega$ of equation $F(x)=0$. 
(ii) There exists $\alpha \geq s_{*}$ such that

$$
\int_{0}^{1} \varphi_{0}\left((1-\theta) \alpha+\theta s_{*}\right) d \theta<1
$$

Set $\Omega_{1}=U\left[x_{0}, \alpha\right] \cap \Omega$. Then, $x^{*}$ is unique in $\Omega_{1}$.

Proof. Let $w^{*} \in \Omega_{1}$ with $F\left(w^{*}\right)=0$. Set $M=\int_{0}^{1} F^{\prime}\left(w^{*}+\theta\left(x^{*}-w^{*}\right)\right) d \theta$. Then, in view of (A2), we obtain in turn that

$$
\begin{aligned}
\left\|F^{\prime}\left(x_{0}\right)^{-1}\left(M-F^{\prime}\left(x_{0}\right)\right)\right\| & \leq \int_{0}^{1} \varphi_{0}\left((1-\theta)\left\|w^{*}-x_{0}\right\|+\theta\left\|x_{*}-x_{0}\right\|\right) d \theta \\
& \leq \int_{0}^{1} \varphi_{0}\left((1-\theta) \alpha+\theta s_{*}\right) d \theta<1,
\end{aligned}
$$

so $w^{*}=x_{*}$ follows from the invertability of $M$ and the estimate $M\left(x_{*}-w^{*}\right)=F\left(x_{*}\right)-$ $F\left(w^{*}\right)=0-0=0$.

\section{Examples}

We present examples to further justify the theoretical results.

Example 1. Consider

$$
\psi(t)=b_{0} t+b_{1}+b_{2} \sin b_{3} t, t_{0}=0,
$$

where $b_{j}, j=0,1,2,3$ are parameters. Then, clearly for $b_{3}$ large and $b_{2}$ small, $\frac{L_{0}}{L_{1}}$ can be small (arbitrarily). Notice that as $\frac{L_{0}}{L_{1}} \longrightarrow 0, \frac{T}{T_{1}} \longrightarrow 0$.

Example 2. If $E_{1}=E_{2}=\mathbb{R}, x_{0}=1$ and $\Omega=U[1,1-p]$ for $p \in\left(0, \frac{2}{3}\right)$, define polynomial $\psi$ on $\Omega$ as

$$
\psi(t)=t^{3}-p .
$$

If we consider case 1 of Newton's method, then, we obtain $L_{0}=3-p, L=L_{1}=2(2-p)$ and $\mu=\frac{1}{3}(1-p)$. But then, $T_{1}>\frac{1}{2}$ for all $p \in\left(0, \frac{1}{2}\right)$. So, Theorem 5.2 in [14] cannot assure convergence. However, we have $T \leq \frac{1}{2}$ for all $p \in I=\left[0.4271907643, \frac{1}{2}\right)$. Hence, our result guarantees convergence to $x^{*}=\sqrt[3]{p}$ as long as $p \in I$.

Example 3. Let $E_{1}=E_{2}=H([0,1])$ the domain of functions given on $[0,1]$ which are continuous. We consider the max-norm. Choose $\Omega=U(0, d), d>1$. Define $G$ on $\Omega$ be

$$
G(x)(s)=x(s)-w(s)-\delta \int_{0}^{1} N(s, t) x^{3}(t) d t
$$

$x \in E_{1}, s \in[0,1], w \in E_{1}$ is given, $\delta$ is a parameter and $N$ is the Green's kernel given by

$$
N\left(b_{2}, b_{1}\right)= \begin{cases}\left(1-b_{2}\right) b_{1}, & b_{1} \leq b_{2} \\ b_{2}\left(1-b_{1}\right), & b_{2} \leq b_{1}\end{cases}
$$

By (50), we have

$$
\left(G^{\prime}(x)(z)\right)(s)=z(s)-3 \delta \int_{0}^{1} N(s, t) x^{2}(t) z(t) d t,
$$

$t \in E_{1}, s \in[0,1]$. Consider $x_{0}(s)=w(s)=1$ and $|\delta|<\frac{8}{3}$. We get

$$
\begin{gathered}
\left\|I-G^{\prime}\left(x_{0}\right)\right\|<\frac{3}{8}|\delta|, G^{\prime}\left(x_{0}\right)^{-1} \in L\left(E_{2}, E_{1}\right), \\
\left\|F^{\prime}\left(x_{0}\right)^{-1}\right\| \leq \frac{8}{8-3|\delta|}, \mu=\frac{|\delta|}{8-3|\delta|}, L_{0}=\frac{12|\delta|}{8-3|\delta|},
\end{gathered}
$$


and $L_{1}=L=\frac{6 \mu|\delta|}{8-3|\delta|}$.

In Table 1 that follows we have listed the results on the convergence criteria for various values of the parameter involved.

Table 1. Comparison table of criteria.

\begin{tabular}{cccc}
\hline $\boldsymbol{\mu}$ & $\delta^{*}$ & $\boldsymbol{T}_{\mathbf{1}}$ & $\boldsymbol{T}$ \\
\hline 2.09899 & 0.9976613778 & 1.007515200 & 0.9639223786 \\
2.19897 & 0.9831766058 & 1.055505600 & 0.9678118280 \\
2.29597 & 0.9698185659 & 1.102065600 & 0.9715205068 \\
3.095467 & 0.87963113211 & 1.485824160 & 1.000082409 \\
\hline
\end{tabular}

Example 4. Let $E_{1}, E_{2}$, and $\Omega$ be as in the Example 3. It is well known that the boundary value problem [16]

$$
\begin{gathered}
\psi(0)=0,(1)=1, \\
\psi^{\prime \prime}=-\psi-\ell \psi^{2}
\end{gathered}
$$

can be presented as a Hammerstein-like nonlinear integral equation [12]

$$
\psi(s)=s+\int_{0}^{1} K(s, t)\left(\psi^{3}(t)+\ell \psi^{2}(t)\right) d t
$$

for $\ell$ being a parameter, consider $F: \Omega \longrightarrow E_{2}$ given by

$$
[F(x)](s)=x(s)-s-\int_{0}^{1} K(s, t)\left(x^{3}(t)+\ell x^{2}(t)\right) d t .
$$

Choose $\psi_{0}(s)=s$ and $\Omega=U\left(\psi_{0}, \rho_{0}\right)$. Then, clearly $U\left(\psi_{0}, \rho_{0}\right) \subset U\left(0, \rho_{0}+1\right)$, since $\left\|\psi_{0}\right\|=1$. Suppose $2 \ell<5$. Then, conditions $(A)$ are satisfied for

$$
L_{0}=\frac{2 \ell+3 \rho_{0}+6}{8}, L_{1}=L=\frac{\ell+6 \rho_{0}+3}{4}
$$

and $\mu=\frac{1+\ell}{5-2 \ell}$. Notice that $L_{0}<L_{1}$.

\section{Conclusions}

Two different techniques and a new domain $D$ included in the original one are introduced. This change in the analysis gives a finer convergence with no additional conditions.

Author Contributions: Conceptualization, S.R., C.I.A., I.K.A. and S.G.; methodology, S.R., C.I.A., I.K.A. and S.G.; software, S.R., C.I.A., I.K.A. and S.G.; validation, S.R., C.I.A., I.K.A. and S.G.; formal analysis, S.R., C.I.A., I.K.A. and S.G.; investigation, S.R., C.I.A., I.K.A. and S.G.; resources, S.R., C.I.A., I.K.A. and S.G.; data curation, S.R., C.I.A., I.K.A. and S.G.; writing-original draft preparation, S.R., C.I.A., I.K.A. and S.G.; writing-review and editing, S.R., C.I.A., I.K.A. and S.G.; visualization, S.R., C.I.A., I.K.A. and S.G.; supervision, S.R., C.I.A., I.K.A. and S.G. project administration, S.R., C.I.A., I.K.A. and S.G.; funding acquisition, S.R., C.I.A., I.K.A. and S.G. All authors have read and agreed to the published version of the manuscript.

Funding: This research received no external funding.

Institutional Review Board Statement: Not applicable.

Informed Consent Statement: Not applicable.

Data Availability Statement: Not applicable.

Conflicts of Interest: The authors declare no conflict of interest. 


\section{References}

1. Argyros, I.K. On the Newton-Kantorovich hypothesis for solving equations. J. Comput. Math. 2004, 169, 315-332. [CrossRef]

2. Argyros, I.K. Convergence and Applications of Newton-Type Iterations; Springer: Berlin/Heidelberg, Germany, 2008.

3. Argyros, I.K.; Hilout, S. Weaker conditions for the convergence of Newton's method. J. Complex. 2012, 28, 364-387. [CrossRef]

4. Argyros, I.K.; Magréñan, A.A. A Contemporary Study of Iterative Methods; Elsevier (Academic Press): New York, NY, USA, 2018.

5. Kantorovich, L.V.; Akilov, G.P. Functional Analysis; Pergamon Press: Oxford, UK, 1982.

6. Nashed, M.Z.; Chen, X. Convergence of Newton-like methods for singular operator equations using outer inverses. Numer. Math. 1993, 66, 235-257. [CrossRef]

7. Ortega, L.M.; Rheinboldt, W.C. Iterative Solution of Nonlinear Equations in Several Variables; Academic Press: New York, NY, USA, 1970.

8. Zabrejko, P.P.; Nguen, D.F. The majorant method in the theory of Newton-Kantorovich approximations and the Pták error estimates. Numer. Funct. Anal. Optim. 1987, 9, 671-684. [CrossRef]

9. Argyros, I.K. Computational Theory of Iterative Methods; Chui, C.K., Wuytack, L., Eds.; Series: Studies in Computational Mathematics 15; Elsevier Publishing Co.: New York, NY, USA, 2007.

10. Argyros, I.K.; Hilout, S. On an improved convergence analysis of Newton's method. Appl. Math. Comput. 2013, 225, 372-386. [CrossRef]

11. Behl, R.; Maroju, P.; Martinez, E.; Singh, S. A study of the local convergence of a fifth order iterative method. Indian J. Pure Appl. Math. 2020, 51, 439-455.

12. Ezquerro, J.A.; Hernandez, M.A. Newton's Method: An Updated Approach of Kantorovich's Theory; Birkhäuser: Cham Switzerland, 2018.

13. Magréñan,A. A.; Gutiérrez, J.M. Real dynamics for damped Newton's method applied to cubic polynomials. J. Comput. Appl. Math. 2015, 275, 527-538. [CrossRef]

14. Potra, F.A.; Pták, V. Nondiscrete Induction and Iterative Processes; Research Notes in Mathematics, 103; Pitman Advanced Publishing Program: Boston, MA, USA, 1984.

15. Proinov, P.D. New general convergence theory for iterative processes and its applications to Newton-Kantorovich type theorems. J. Complex. 2010, 26, 3-42. [CrossRef]

16. Shakhno, S.M.; Iakymchuk, R.P.; Yarmola, H.P. Convergence analysis of a two step method for the nonlinear squares problem with decomposition of operator. J. Numer. Appl. Math. 2018, 128, 82-95.

17. Sharma, J.R.; Guha, R.K.; Sharma, R. An efficient fourth order weighted-Newton method for systems of nonlinear equations. Numer. Algorithms 2013, 62, 307-323. [CrossRef]

18. Verma, R. New Trends in Fractional Programming; Nova Science Publisher: New York, NY, USA, 2019.

19. Rheinboldt, W.C. An Adaptive Continuation Process of Solving Systems of Nonlinear Equations; Polish Academy of Science, Banach Center Publications: Greifswald, Germany, 1978; Volume 3, pp. 129-142.

20. Soleymani, F.; Lotfi, T.; Bakhtiari, P. A multi-step class of iterative methods for nonlinear systems. Optim. Lett. 2014, 8, 1001-1015. [CrossRef]

21. Traub, J.F. Iterative Methods for the Solution of Equations; Prentice Hall: Upper Saddle River, NJ, USA, 1964.

22. Traub, J.F.; Werschulz, A.G. Complexity and Information, Lezioni Lince; Lincei Lectures; Cambridge University Press: Cambridge, UK, 1998; pp. xii+139. ISBN 0-521-48506-1. 